\title{
Prediction of PSA Progression in Castration-Resistant Prostate Cancer Based on Treatment-Associated Change in Tumor Burden Quantified by ${ }^{18}$ F-Fluorocholine PET/CT
}

\author{
Joohee Lee ${ }^{1,2}$, Miles M. Sato ${ }^{1}$, Marc N. Coel ${ }^{3}$, Kyung-Han Lee ${ }^{2}$, and Sandi A. Kwee ${ }^{3,4}$ \\ ${ }^{1}$ Oncology Research Program, Queen's Medical Center, Honolulu, Hawaii; ${ }^{2}$ Department of Nuclear Medicine, Samsung Medical \\ Center, Sungkyunkwan University School of Medicine, Seoul, Republic of Korea; ${ }^{3}$ Hamamatsu/Queen's PET Imaging Center, Queen's \\ Medical Center, Honolulu, Hawaii; and ${ }^{4}$ Department of Medicine, John A. Burns School of Medicine, University of Hawaii, Honolulu, \\ Hawaii
}

\begin{abstract}
Measurements of metabolically active tumor volume (MATV) can be applied to ${ }^{18} \mathrm{~F}$-fluorocholine PET/CT to quantify whole-body tumor burden. This study evaluated the serial application of these measurements as systemic treatment response markers and predictors of disease progression in patients with castration-resistant prostate cancer (CRPC). Methods: Forty-two patients completed sequential ${ }^{18} \mathrm{~F}$-fluorocholine PET/CT scans before and 1-3 mo after starting treatment for CRPC. Whole-body tumor segmentation was applied to determine net MATV from each scan. Changes in net MATV were evaluated as predictors of time to prostate-specific antigen (PSA) progression by Kaplan-Meier and proportional hazards regression analysis. Results: Treatments consisted of chemotherapy in 16 patients, antiandrogens in 19 patients, ${ }^{223}$ Ra-dichloride in 5 patients, and sipuleucel-T in 2 patients. A significant MATV response (defined as $a \geq 30 \%$ decrease in net MATV) was observed in 20 patients on the basis of in-treatment PET/CT performed an average of $51 \mathrm{~d}$ (median, $49 \mathrm{~d}$ ) into treatment. Significantly longer times to PSA progression were observed in patients who exhibited an MATV response (418 d vs. $116 \mathrm{~d}, P=0.0067$ ). MATV response was associated with a hazard ratio of $0.246(P=0.0113)$ for PSA progression, which remained significant when adjusted for treatment type. Conclusion: Significant changes in whole-body tumor burden can be measured on ${ }^{18} \mathrm{~F}$-fluorocholine PET/CT over the course of contemporary treatments for CRPC. In this study, these changes were found to be predictive of PSA progression as a potential surrogate marker of treatment outcome. Because ${ }^{18} \mathrm{~F}$-fluorocholine PET/CT can also be used for localizing resistant tumors, this modality can potentially complement other measures of response in the precision management of advanced prostate cancer.
\end{abstract}

Key Words: fluorocholine; PET/CT; castrate resistant prostate cancer; treatment response; tumor volume

J Nucl Med 2016; 57:1058-1064

DOI: 10.2967/jnumed.115.169177

\footnotetext{
Received Nov. 24, 2015; revision accepted Jan. 26, 2016.

For correspondence or reprints contact: Sandi A. Kwee, PET Imaging, Queen's Medical Center, 1301 Punchbowl St., First Floor, Honolulu, Hawaii 96813.

E-mail: kwee@hawaii.edu

Published online Feb. 16, 2016.

COPYRIGHT (c) 2016 by the Society of Nuclear Medicine and Molecular Imaging, Inc.
}

$\mathbf{P}$ worldwide, and mortality from this disease is increasing in some regions, including Eastern Europe, Russia, China, and Korea (1). Over two thirds of prostate cancer cases are now diagnosed in developed countries, where patients are typically identified at an early stage and treated first with prostatectomy or radiation therapy $(1,2)$. For patients who present with metastatic disease or in whom the disease recurs after these primary treatments, hormonal therapy is readily available, delaying disease progression until the development of castration-resistant prostate cancer (CRPC) (2).

Effective agents for treating CRPC have fortunately expanded over the past decade to include chemotherapy, as well as advanced antiandrogens, immunotherapy, and, recently, radiopharmaceuticals (2). However, these agents may improve survival by only weeks or months, and precision management of CRPC remains difficult because of the scarcity of tools for ascertaining tumor response in real time $(3,4)$. Although posttreatment declines in the level of prostate-specific antigen (PSA) have been used in some prostate cancer clinical trials as a surrogate marker of response (5), these PSA-response measures have accounted for only a limited degree of the variation in treatment outcome in CRPC clinical trials $(3,6)$. Because treatments for CRPC can also lead to clonal selection of tumors with downregulated PSA expression (7), biomarkers that can complement PSA will be needed not only to advance drug development but also to support the evolution of precision medicine in prostate cancer.

Although conventional imaging modalities (e.g., CT, MRI, and bone scintigraphy) are useful for oncologic staging in prostate cancer, they are imperfect as treatment response biomarkers for CRPC because of their inherent limitations in quantifying therapeutic effects along two dominant metastatic routes in CRPC: the skeletal system and the lymphatic system (8). Molecular imaging, despite being more cumbersome than conventional imaging or serology, can reproducibly assess the skeletal system and other clinically relevant sites of disease activity in CRPC (4) and thus can potentially complement other biomarkers while also helping to direct sequential treatments toward pockets of resistant disease.

One molecular imaging agent, ${ }^{18} \mathrm{~F}$-fluorocholine, enables in vivo characterization of choline metabolism using PET/CT. It has shown greater tumor avidity than ${ }^{18} \mathrm{~F}-\mathrm{FDG}$ in both androgen-dependent and androgen-independent prostate cancer (9). Tissue uptake of ${ }^{18} \mathrm{~F}$ fluorocholine mirrors the initial steps of tissue phosphatidylcholine synthesis, which is often high in CRPC tumors but can diminish on 
exposure to traditional chemotherapeutic agents such as docetaxel and cabazitaxel (10) and newer agents such as abiraterone (11) and ${ }^{223}$ Ra-dichloride (12). Recently, estimates of tumor burden based on net metabolically active tumor volume (MATV) measurements from ${ }^{18} \mathrm{~F}$-fluorocholine PET/CT were shown to have prognostic value in patients with CRPC (13). The objective of the present study was to determine whether treatment-associated changes in these measurements on sequential ${ }^{18} \mathrm{~F}$-fluorocholine PET/CT scans are predictive of treatment outcome in CRPC.

\section{MATERIALS AND METHODS}

\section{Patients}

This prospective clinical research study was conducted in accordance with the Declaration of Helsinki and its amendments. Forty-five patients (18 prescribed docetaxel-based chemotherapy, 19 prescribed antiandrogens, 6 prescribed ${ }^{223}$ Ra-dichloride, and 2 prescribed sipuleucel-T) were enrolled after consenting in writing to participate in this institutional review board-approved study. Study eligibility was based on age over $18 \mathrm{y}$, an initial histopathologic diagnosis of prostate cancer, fulfillment of clinical criteria for CRPC as defined by 2 rising PSA measurements of $2.0 \mathrm{ng} / \mathrm{mL}$ or higher while on complete androgen blockade for longer than 3 mo, planned treatment for CRPC under the supervision of a medical oncologist or urologist, and life expectancy of more than 12 wk. Patients who could not tolerate PET/CT imaging and those with other malignancies diagnosed in the past $3 \mathrm{y}$, except for basal cell carcinoma or superficial transitional cell carcinoma of the bladder, were excluded.

Patients were treated in a community setting, with all enrollment and clinical follow-up performed between August 2009 and August 2015. All patients underwent baseline ${ }^{18} \mathrm{~F}$-fluorocholine PET/CT before starting treatment for CRPC. A second in-treatment ${ }^{18} \mathrm{~F}$-fluorocholine PET/CT scan was scheduled before the midpoint of treatment at approximately 1-3 mo, corresponding to clinic visits after the second chemotherapy treatment cycle, antiandrogen therapy follow-up, or the second dose of ${ }^{223} \mathrm{Ra}$-dichloride. All treatment decisions were made independently of the study by a medical oncologist or urologist.

\section{Radiopharmaceutical Synthesis}

An 11-MeV cyclotron (RDS 111; Siemens Medical Solutions) was used to produce ${ }^{18} \mathrm{~F}$. The radiotracer was synthesized by fluorination of ditosylmethane followed by alkylation of the intermediate with dimethylethanolamine using an automated chemical process control unit (CTI/ Siemens) (14). Each radiopharmaceutical batch passed standard assays for radiochemical purity, radionuclide identity, lack of pyrogenicity, and chemical purity before use. The final radiochemical purity was $99 \%$.

\section{PET/CT Imaging}

All patients refrained from eating and drinking for at least $3 \mathrm{~h}$ before undergoing PET/CT. Imaging was performed using a Gemini TF-64 PET/CT scanner (Philips Healthcare) beginning with CT scanning from the mid thigh to the skull with the patient supine. The 64-channel helical CT scanning parameters were $120 \mathrm{kV}, 50 \mathrm{~mA} /$ slice, a rotation time of $0.75 \mathrm{~s}$, and slice thickness and interval of $5.0 \mathrm{~mm}$. No iodinated contrast agents were given. At approximately $10-15 \mathrm{~min}$ after the intravenous injection of a $2.6 \mathrm{MBq} / \mathrm{kg}(0.07 \mathrm{mCi} / \mathrm{kg})$ dose of ${ }^{18} \mathrm{~F}$-fluorocholine, sequential static emission scans were obtained from the mid thigh to the skull at 2 min per section. Images were reconstructed using a vendor-supplied maximum-likelihood expectation maximization process, with CT data applied for attenuation correction.

\section{Image Analysis}

Lesion segmentation and MATV measurements were performed as described in a previous publication (13). Briefly, the presence of tumors as seen on ${ }^{18} \mathrm{~F}$-fluorocholine PET/CT images was classified by consen- sus between an interpreter with significant experience and an interpreter with $3 \mathrm{wk}$ of training in ${ }^{18} \mathrm{~F}$-fluorocholine PET/CT interpretation. Lesions were classified according to their anatomic location in the following categories: the prostate gland, a visceral organ, a lymph node, or the skeleton. Only lesions with an $\mathrm{SUV}_{\text {max }}$ of 3.0 or greater (exceeding 2 SDs above the normal-marrow SUV) were included for MATV measurement. SUV was calculated as measured voxel activity divided by injected radioactivity normalized to body weight. The MATV for each lesion was computed using a semiautomated segmentation algorithm. A volume of interest was generated around the voxel corresponding to the $\mathrm{SUV}_{\text {max }}$ of the lesion, encompassing all contiguous voxels with SUV exceeding $40 \%$ of the lesion $\mathrm{SUV}_{\text {max }}$ based on a previous study (13). A measure of activity distribution within the volume, termed total lesion activity (TLA), was also calculated by taking the product of lesion $\mathrm{SUV}_{\text {mean }}$ and MATV. Measures reflecting the net tumor burden on each scan were defined as the sum of all MATVs (net MATV) and the sum of all TLAs (net TLA). Indices of whole-body tumor response were then calculated as the percentage change in these measures between baseline and in-treatment ${ }^{18} \mathrm{~F}$-fluorocholine PET/CT scans. An MATV $30 \%$ response was defined prospectively as a $30 \%$ or greater decline in net MATV from the pretreatment net MATV, and a TLA $30 \%$ response was defined prospectively as a $30 \%$ or greater decline in net TLA from the pretreatment net TLA.

\section{PSA Measurements}

Clinical PSA levels measured from the start of treatment over the period of follow-up were recorded. Time to PSA progression was calculated as the number of days from the start of treatment to the date of the first PSA test result that represented a $30 \%$ or greater increase from the PSA nadir, confirmed on the basis of repeated PSA measurements. To explore relationships between changes in PSA and changes in tumor indices on ${ }^{18} \mathrm{~F}$-fluorocholine PET/CT, the percentage change in PSA level within $15 \mathrm{wk}$ of starting treatment was calculated, using a $50 \%$ or greater decrease in PSA level as a predefined definition of PSA response based on Prostate Cancer Working Group guidelines (5).

\section{Statistical Analysis}

Kaplan-Meier analysis was used to compare rates of PSA progression in patients stratified by $\mathrm{MATV}_{30 \%}$ response or $\mathrm{TLA}_{30 \%}$ response, with differences in progression curves assessed using the Wilcoxon and log-rank tests. Cox proportional hazards regression was used to evaluate the individual effects of age, baseline pretreatment PSA, early changes in PSA, and type of treatment on time to PSA progression. Multivariate analysis was used in a limited fashion to explore the interactions between indices derived from PET/CT and another variable. Differences between serial measurements were assessed by the Wilcoxon signed-rank test. Unpaired differences were assessed by the Mann-Whitney $U$ test. Differences across categories were assessed by ANOVA using the KruskalWallis test. Correlations were assessed using Spearman correlation. A probability of less than 0.05 was considered statistically significant. All statistical tests were 2-sided and performed using SAS, version 9.4, and JMP Pro, version 11 (SAS Institute Inc.).

\section{RESULTS}

\section{Clinical Characteristics}

Forty-five sequential patients were enrolled in the study. However, 2 patients experienced delays in treatment after the baseline PET/CT scan and were excluded, and one patient was found on baseline PET/CT to have intracranial metastases, leading to cancellation of the planned treatment. Therefore, data from only 42 patients were included in the analysis. The mean, median, and range for clinical follow-up duration were 784, 653, and 180-2,068 d, 
TABLE 1

Patient Characteristics $(n=42)$

\begin{tabular}{|c|c|}
\hline Characteristic & Value \\
\hline Age $(y)$ & $73(70.0-75.2)$ \\
\hline Baseline PSA (ng/mL) & $329.4(12.2-646.7)$ \\
\hline \multicolumn{2}{|l|}{ Treatment type $(n)$} \\
\hline Chemotherapy & 16 \\
\hline Antiandrogen & 19 \\
\hline${ }^{223}$ Ra-dichloride & 5 \\
\hline Sipuleucel-T & 2 \\
\hline \multicolumn{2}{|l|}{$\begin{array}{l}\text { PET tumor burden } \\
\text { measurements }\end{array}$} \\
\hline Baseline net TLA (g) & $2,380.8(706.9-4,054.6)$ \\
\hline Follow-up net TLA (g) & $1,438.9(555.0-2,322.8)$ \\
\hline Baseline net MATV $\left(\mathrm{cm}^{3}\right)$ & $516.0(151.9-880.1)$ \\
\hline Follow-up net MATV $\left(\mathrm{cm}^{3}\right)$ & $356.1(135.7-576.4)$ \\
\hline \multicolumn{2}{|l|}{$\begin{array}{l}\text { Sites of disease on first } \\
\text { PET/second PET }(n)\end{array}$} \\
\hline Bone & $32(76 \%) / 30(71 \%)$ \\
\hline Lymphatics & $19(45 \%) / 15(36 \%)$ \\
\hline Prostate & $13(31 \%) / 12(29 \%)$ \\
\hline Lung and adrenal glands & $3(7 \%) / 3(7 \%)$ \\
\hline
\end{tabular}

Continuous values are expressed as mean followed by $95 \%$ confidence interval in parentheses.

respectively. The clinical characteristics and treatments of the 42 patients are summarized in Table 1 . There were no statistically significant differences in baseline patient characteristics across treatments (Table 2).

\section{PSA Measurements}

Changes in PSA levels measured within 15 wk of treatment initiation ranged from a $100 \%$ decrease to a $1,648 \%$ increase (with a median $8 \%$ decrease). A PSA response based on the Prostate Cancer Working Group definition was noted for 20 of the 42 patients. Percentage change in PSA level correlated moderately with percentage change in net MATV $(r=0.71, P<0.0001)$ and with percentage change in net TLA $(r=0.73, P<0.0001)$. However, an increase in net MATV/TLA coincided with a decrease in PSA level in 5 patients, and a decrease in net MATV/TLA coincided with an increase in PSA level in 3 patients. On followup, PSA progression was identified in 17 patients, with a mean, median, and range for time to progression of 142, 116, and 62-418 d, respectively. Early PSA progression was noted in 2 patients at 62 and $76 \mathrm{~d}$ in accordance with the Prostate Cancer Working Group criteria for treatment discontinuation based on clinical progression (5).

\section{${ }^{18}$ F-Fluorocholine PET/CT Findings}

The respective mean, median, and range were 4, 1, and 0-26 d for the interval from baseline ${ }^{18} \mathrm{~F}$-fluorocholine PET/CT to start of treatment and 51, 49, and 21-98 d for the interval from start of treatment to in-treatment ${ }^{18} \mathrm{~F}$-fluorocholine PET/CT. Twenty patients met the study criteria for an $\mathrm{MATV}_{30 \%}$ response. The same 20 patients also met the study criteria for a $\mathrm{TLA}_{30 \%}$ response. Because the distribution of patients with MATV $_{30 \%}$ responses was the same as the distribution of patients with $\mathrm{TLA}_{30 \%}$ responses, the predictive power of the two indices was the same, and thus in discussing these results we will use the term MATV response generically to refer to either $\mathrm{MATV}_{30 \%}$ response or $\mathrm{TLA}_{30 \%}$ response. The anatomic distributions of lesions detected on the baseline and in-treatment ${ }^{18} \mathrm{~F}$ fluorocholine PET/CT scans are summarized in Table 1. Examples of the changes in tumor activity and MATV that can be quantified on ${ }^{18} \mathrm{~F}$-fluorocholine PET/CT are shown in Figures 1 through 3.

\section{Analysis of PSA Progression}

Time to PSA progression differed significantly between patients with and without an MATV response (median, $418 \mathrm{~d}$ vs. $116 \mathrm{~d}$, respectively, log-rank $P=0.0067$, Wilcoxon $P=0.0138$ ). On univariate analysis, MATV response was associated with a significantly lower risk of PSA progression (hazard ratio, 0.246; $P=$ 0.0113) (Fig. 4), whereas age, baseline PSA level, PSA response, and the distribution of lesions on PET/CT were not significantly associated with PSA progression (Table 3). However, chemotherapy was associated with a relatively lower risk of PSA progression (hazard ratio, $0.309 ; P=0.0333$ ), whereas antiandrogen treatment was associated with a relatively higher risk (hazard ratio, 5.650; $P=0.0236$ ). MATV response remained significant as a predictor of PSA progression on multivariate analysis adjusted for treatment type (Table 3).

\section{DISCUSSION}

Over the past decade, a variety of treatments directed toward different cellular targets and mechanisms has emerged to improve the survival of men with CRPC (2). However, assessing treatment

TABLE 2

Baseline Clinical Parameters According to Treatment Type

\begin{tabular}{|c|c|c|c|c|c|}
\hline \multirow[b]{2}{*}{ Variable } & \multicolumn{4}{|c|}{ Treatment type } & \multirow[b]{2}{*}{$P$} \\
\hline & Antiandrogen $(n=19)$ & Docetaxel $(n=16)$ & ${ }^{223} \mathrm{Ra}(n=5)$ & Sipuleucel-T $(n=2)$ & \\
\hline Age (y) & 75.1 & 69.9 & 75.0 & 64.5 & 0.0789 \\
\hline Baseline PSA (ng/mL) & 293.7 & 331.0 & 577.1 & 36.5 & 0.3460 \\
\hline Baseline net MATV $\left(\mathrm{cm}^{3}\right)$ & 591.2 & 582.5 & 208.7 & 37.8 & 0.8010 \\
\hline Baseline net TLA (g) & $2,691.5$ & $2,768.3$ & 837.3 & 186.5 & 0.8224 \\
\hline \multicolumn{6}{|c|}{$P$ values are by Kruskal-Wallis Test. } \\
\hline
\end{tabular}




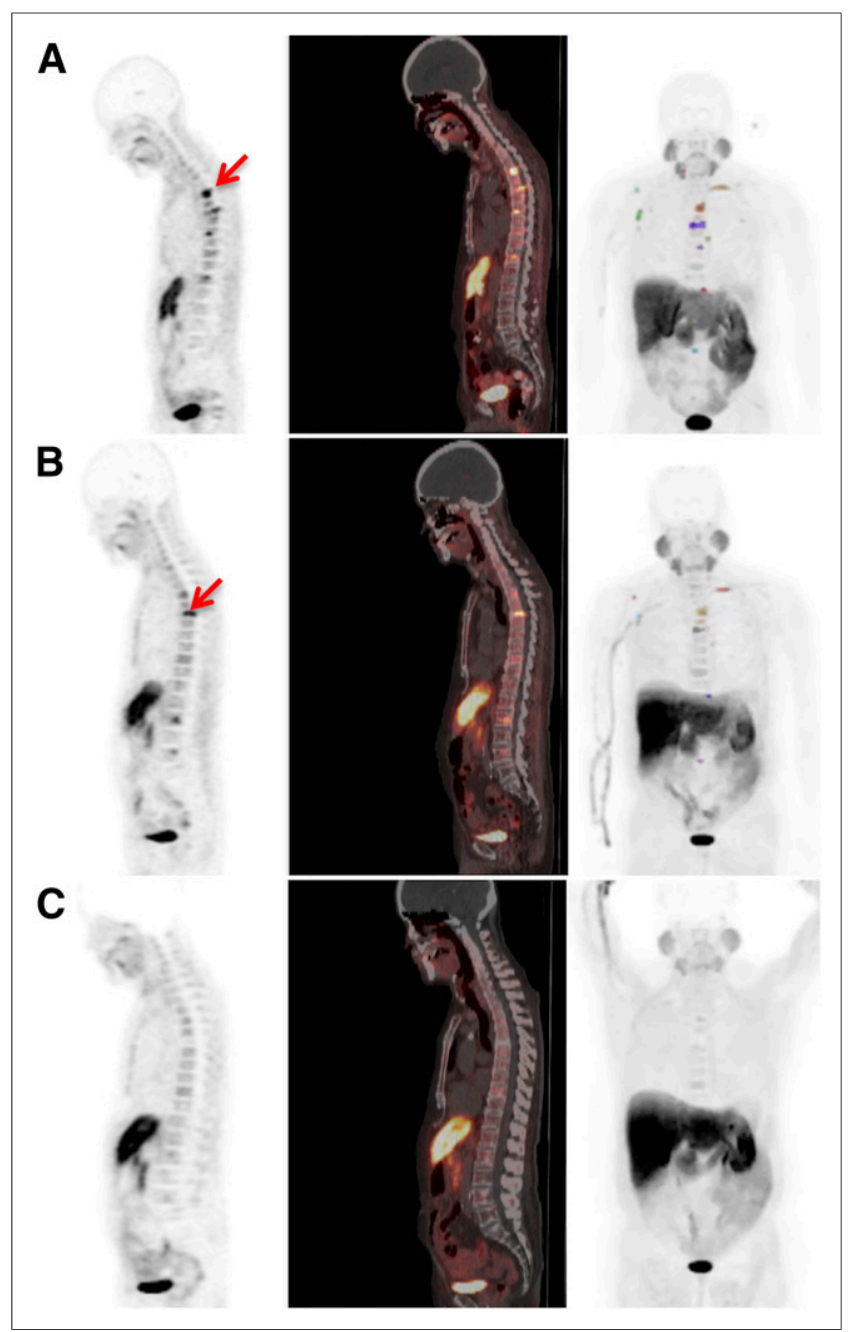

FIGURE 1. Discordance between early MATV response and PSA response, as shown by ${ }^{18} \mathrm{~F}$-fluorocholine PET (left), PET/CT (middle), and maximum-intensity-projection (MIP) (right) images from 74-y-old patient receiving docetaxel. Color indicates MATV contours on MIP images. (A) Multiple bone metastases are evident on pretreatment PET/CT (arrow; net MATV, $42.7 \mathrm{~cm}^{3}$; PSA, $39.3 \mathrm{ng} / \mathrm{mL}$ ). (B) Net MATV decreased after second chemotherapy cycle to $21.6 \mathrm{~cm}^{3}$, whereas PSA increased to $41.7 \mathrm{ng} / \mathrm{mL}$. (C) After sixth chemotherapy cycle, PET/CT demonstrated resolution of abnormal activity (net MATV, $0.0 \mathrm{~cm}^{3}$ ), and PSA level decreased to $8.1 \mathrm{ng} / \mathrm{mL}$.

efficacy in CRPC remains a significant clinical challenge $(3-5,8)$. In the present study, whole-body indices of tumor response derived from ${ }^{18} \mathrm{~F}$-fluorocholine PET/CT were evaluated as therapeutic predictors in patients undergoing contemporary management of CRPC. This study found that decreases in net MATV observed early in the course of treatments for CRPC were associated with significantly longer times to PSA progression independently of other clinical characteristics such as the type of treatment given. This observation supports ${ }^{8} \mathrm{~F}$-fluorocholine PET/CT as a potential independent predictor of treatment outcome in CRPC. ${ }^{18} \mathrm{~F}$-fluorocholine has been extensively studied for prostate cancer, and our study contributes to data supporting its usefulness across a variety of clinical applications that require accurate determination of metastatic spread (15). Previous ${ }^{18} \mathrm{~F}$-fluorocholine PET/CT studies of CRPC have documented the significant effect of treatment with antiandrogens and ${ }^{223} \mathrm{Ra}$ on disease pattern $(12,16)$. Our study provides further examples by documenting such effects for docetaxel and sipuleucel-T as well.

Time to PSA progression was the main endpoint of this study for evaluating MATV response because increases in PSA level after the posttreatment nadir (i.e., PSA progression) have proven more reliable in predicting clinical outcomes in CRPC than are acute changes in the PSA level after treatment initiation (i.e., PSA response) (6,17-19). Nonetheless, tumor metabolism depicted by ${ }^{18} \mathrm{~F}$-fluorocholine PET/CT was also evaluated in relation to early changes in PSA level. Early changes in PSA level were found to correlate moderately with changes in tumor activity quantified on ${ }^{18}$ F-fluorocholine PET/CT. These results were comparable to those of a previous study evaluating ${ }^{11} \mathrm{C}$-choline for measuring therapeutic response to docetaxel in CRPC (20). Like this previous study, our study found that decreases in tumor activity on ${ }^{18} \mathrm{~F}$-fluorocholine PET/CT did not necessarily coincide with decreases in PSA

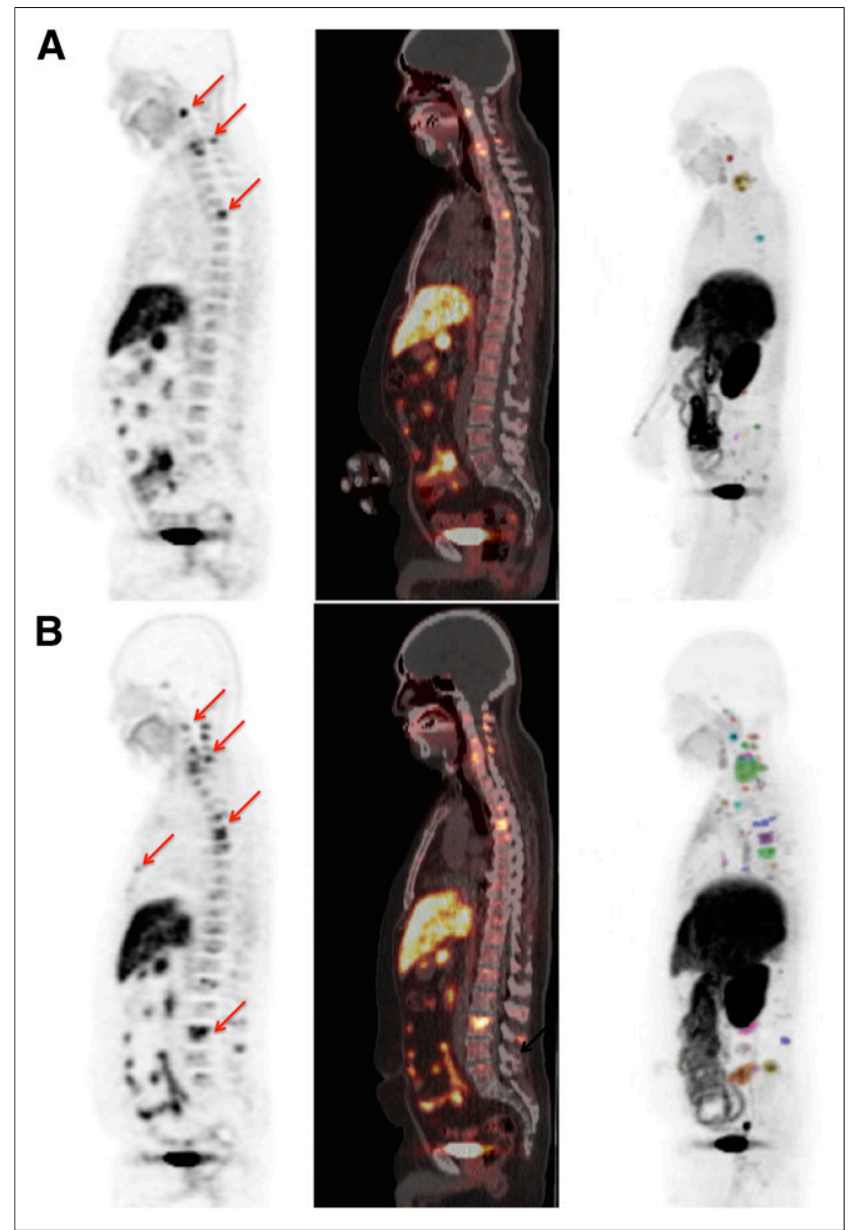

FIGURE 2. Cancer progression reflected by increasing net MATV as shown on ${ }^{18} \mathrm{~F}$-fluorocholine PET (left), PET/CT (middle), and maximumintensity-projection (MIP) (right) images from 65-y-old patient receiving sipuleucel-T. Color indicates MATV contours on MIP images. (A) Pretreatment PET/CT shows hyperactive vertebral metastases (arrows; net MATV, $42.9 \mathrm{~cm}^{3}$; PSA, $38.0 \mathrm{ng} / \mathrm{mL}$ ). (B) PET/CT image obtained $36 \mathrm{~d}$ after initiation of treatment demonstrates increasing activity and new lesions in sternum and lumbar spine (arrows; net MATV, $338.4 \mathrm{~cm}^{3}$; PSA, $46.7 \mathrm{ng} / \mathrm{mL}$ ). PSA level after $4 \mathrm{mo}$ increased to $241.4 \mathrm{ng} / \mathrm{mL}$. 


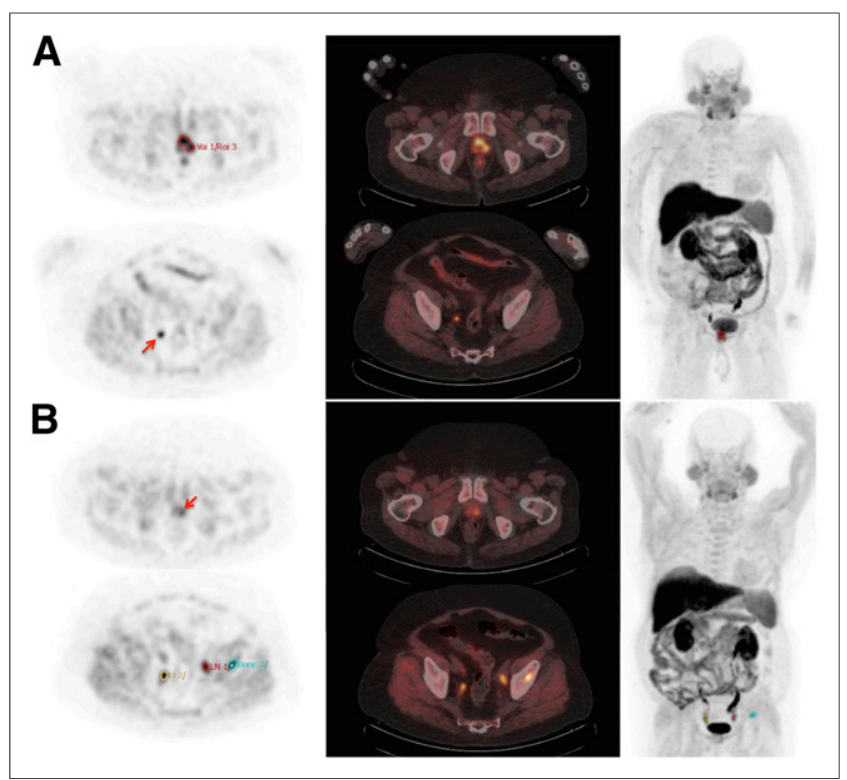

FIGURE 3. Heterogeneous response as shown on serial ${ }^{18} \mathrm{~F}$-fluorocholine PET (left), PET/CT (middle), and maximum-intensity-projection (MIP) (right) images from 64-y-old patient receiving sipuleucel-T. (A) Pretreatment images show abnormal activity in prostate gland (red contour in upper image). (B) PET/CT images obtained $60 \mathrm{~d}$ after start of treatment show significant decline in prostatic activity (arrow in upper image) but new abnormal activity in left iliac lymph node and ilium (red and blue contours in lower image). Net MATV increased from 32.5 to $34.0 \mathrm{~cm}^{3}$, and PSA increased from 35.0 to $47.9 \mathrm{ng} / \mathrm{mL}$. PSA progression was confirmed 3 mo later $(65.1 \mathrm{ng} / \mathrm{mL})$. Excreted tracer in right ureter (arrow in lower image of $A$ and yellow contour in lower image of B) was not mistaken for lesion.

level and, in fact, coincided with increasing PSA levels in some cases (Fig. 1). The limitations of acute PSA changes as a response measure in CRPC have been well described $(3,6)$. For example, acute rises in PSA level (PSA flares) may occur in over $10 \%$ of CRPC patients receiving docetaxel and, although not predictive of treatment outcome, can confound decisions on whether to continue treatment (17). Conversely, declines in PSA level may also be misleading, since some treatments can inhibit PSA production independently of their tumoricidal effects (7). Although we show in Figure 1 a case in which PET/CT and PSA measurements proved discordant despite being performed within $24 \mathrm{~h}$ of each other, the average interval between in-treatment follow-up PET/CT and follow-up PSA measurements in the present study was $8 \mathrm{~d}$. Therefore, additional studies will be needed to further investigate the treatment-associated changes in tumor metabolic activity underlying fluctuations in PSA production in CRPC.

The present study evaluated indices of treatment response that incorporated every tumor lesion identified on ${ }^{18} \mathrm{~F}$-fluorocholine PET/CT. In contrast, other studies have evaluated predictive indices derived from a fixed number of target lesions $(11,21)$. This latter approach may be simpler and quicker to perform than whole-body tumor assessments. However, because metastases can vary in morphology, immune phenotype, and genotype within the same patient (22), assessments of treatment response reflecting only a limited number of lesions may be misleading. The feasibility and reproducibility of whole-body- and target-lesion-based approaches for assessing treatment response deserve further comparisons in a clinical trial setting.
There are several promising investigational ${ }^{18} \mathrm{~F}$-labeled tracers for prostate cancer imaging on the horizon, such as the prostate-specific membrane antigen ligands and trans-1-amino-3- ${ }^{18} \mathrm{~F}$-fluorocyclobutanecarboxylic acid $(4,23,24)$, as well as sodium ${ }^{18}$ F-fluoride, which is commercially available in many areas (25). Because of the high lesion-to-background uptake ratios associated with these tracers, they should also work for whole-body quantification of tumor burden based on MATV measurements. However, each tracer warrants its own evaluation as a treatment biomarker because the expression of some imaging targets may not necessarily change in response to treatment in a manner consistent with other targets (7). Because one of the most extensively studied PET techniques for prostate cancer imaging is ${ }^{18} \mathrm{~F}$-fluorocholine PET/CT (15), it was a good platform for developing methods to assess tumor response in CRPC using whole-body MATV measurements.

This study, having been performed at a single institution and on a limited number of patients, had certain limitations. MATV calculations were based on voxel SUV measurements and thus depended on the PET scanner calibration, imaging protocol, and image reconstruction method used by the institution. Additional efforts are required not only to validate the concept of quantifying whole-body tumor response with ${ }^{18} \mathrm{~F}$-fluorocholine PET/CT but also to generalize the methods supporting this approach. Furthermore, the fact that our study enrolled patients without regard to treatment type introduced potential treatment-related bias. However, no significant associations between treatment type and clinical parameters were noted (Table 2).

Previously, tumor burden measured on ${ }^{18} \mathrm{~F}$-fluorocholine PET/ CT was found to be significantly associated with overall survival in CRPC even after adjustment for treatments administered after the patients were imaged (13). This finding implies either that survival in CRPC patients is influenced largely by the extent of disease to be treated or that individual treatments have a relatively small impact on survival. However, because there are now multiple dissimilar treatments for CRPC that may impact specific manifestations of disease differently, it may be possible to judiciously base treatment selection on an individual patient's disease pattern in order to compound the survival benefit of sequential treatments. The findings of the present study across the current spectrum of

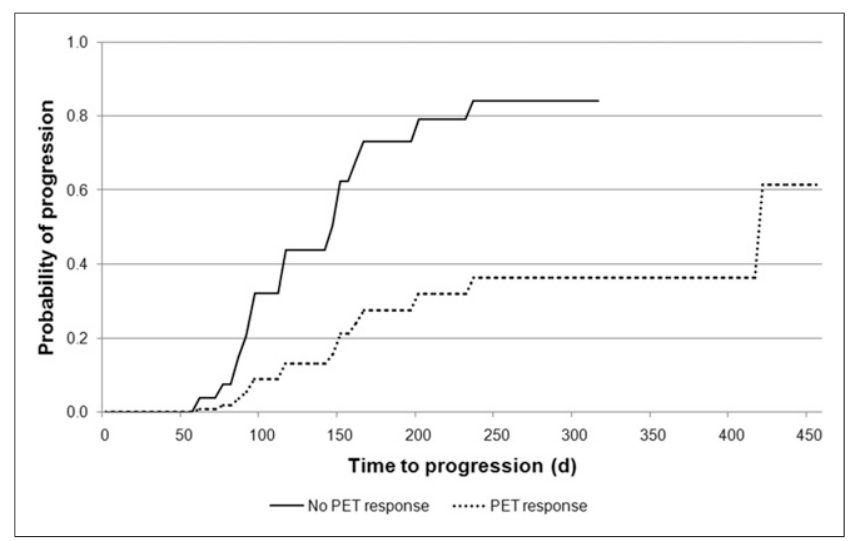

FIGURE 4. Probability of PSA progression over time based on MATV response. Median time to PSA progression was $418 \mathrm{~d}$ in MATV responders and $116 \mathrm{~d}$ in MATV nonresponders. Both early and late differences in time to PSA progression were statistically significant on Kaplan-Meier analysis (Wilcoxon $P=0.0138$ and log-rank $P=$ 0.0067). 
TABLE 3

Proportional Hazards Regression Analysis of Time to PSA Progression

\begin{tabular}{|c|c|c|c|}
\hline Variable & Hazard ratio & $95 \%$ confidence limits & $P$ \\
\hline \multicolumn{4}{|l|}{ Clinical parameters } \\
\hline Age $>70 y$ & 0.756 & $0.274-2.078$ & 0.5882 \\
\hline PSA level before treatment & 1.050 & $0.778-1.422$ & 0.7424 \\
\hline $\mathrm{PSA}$ response at $\leq 15 \mathrm{wk}$ & 0.383 & $0.125-1.173$ & 0.0944 \\
\hline \multicolumn{4}{|l|}{ PET parameters } \\
\hline MATV $_{30 \%}$ response & 0.246 & $0.083-0.728$ & 0.0113 \\
\hline $\mathrm{TLA}_{30 \%}$ response & 0.246 & $0.083-0.728$ & 0.0113 \\
\hline \multicolumn{4}{|l|}{ Lesion pattern on PET } \\
\hline Bone involvement & 1.773 & $0.617-5.092$ & 0.2902 \\
\hline Lymph node involvement & 0.967 & $0.368-2.538$ & 0.9452 \\
\hline Prostate involvement & 0.870 & $0.321-2.357$ & 0.7857 \\
\hline Visceral involvement & 2.219 & $0.273-18.05$ & 0.4584 \\
\hline \multicolumn{4}{|l|}{ Treatment type } \\
\hline Chemotherapy vs. other & 0.309 & $0.105-0.906$ & 0.0333 \\
\hline Antiandrogen vs. other & 5.650 & $1.2715-25.1$ & 0.0236 \\
\hline \multicolumn{4}{|l|}{ Multivariate analysis } \\
\hline Age-adjusted PET response & 0.227 & $0.074-0.698$ & 0.0100 \\
\hline Chemotherapy-adjusted PET response & 0.298 & $0.097-0.922$ & 0.0366 \\
\hline Antiandrogen treatment-adjusted PET response & 0.298 & $0.096-0.930$ & 0.0380 \\
\hline
\end{tabular}

treatments for CRPC suggest that ${ }^{18} \mathrm{~F}$-fluorocholine PET/CT may be able to support such a strategy.

\section{CONCLUSION}

In this study, significant changes in whole-body tumor burden measured on ${ }^{18} \mathrm{~F}$-fluorocholine PET/CT early in the course of contemporary treatments for CRPC were found to be predictive of PSA progression. The growing selection of treatments for CRPC and the feasibility of applying ${ }^{18} \mathrm{~F}$-fluorocholine PET/ CT for measuring changes in tumor burden after those treatments support further clinical evaluation of this imaging approach in the management of advanced prostate cancer.

\section{DISCLOSURE}

The costs of publication of this article were defrayed in part by the payment of page charges. Therefore, and solely to indicate this fact, this article is hereby marked "advertisement" in accordance with 18 USC section 1734. The U.S. National Institutes of Health supported this work under National Cancer Institute grant R21CA139687-02. Patients receiving antiandrogen treatments were enrolled under clinicaltrials.gov study NCT00928174, and all subsequent patients were enrolled under study NCT00928252. Additional funding was provided by Bayer Healthcare to support the enrollment of ${ }^{223} \mathrm{Ra}$-treated patients through an InvestigatorInitiated Studies Program. No other potential conflict of interest relevant to this article was reported.

\section{REFERENCES}

1. Center MM, Jemal A, Lortet-Tieulent J, et al. International variation in prostate cancer incidence and mortality rates. Eur Urol. 2012;61:1079-1092.
2. Mohler JL, Kantoff PW, Armstrong AJ, et al. Prostate cancer, version 2.2014. J Natl Compr Canc Netw. 2014;12:686-718.

3. Armstrong AJ, Eisenberger MA, Halabi S, et al. Biomarkers in the management and treatment of men with metastatic castration-resistant prostate cancer. Eur Urol. 2012;61:549-559.

4. Picchio M, Mapelli P, Panebianco V, et al. Imaging biomarkers in prostate cancer: role of PET/CT and MRI. Eur J Nucl Med Mol Imaging. 2015;42:644655.

5. Scher HI, Halabi S, Tannock I, et al. Design and end points of clinical trials for patients with progressive prostate cancer and castrate levels of testosterone: recommendations of the Prostate Cancer Clinical Trials Working Group. J Clin Oncol. 2008;26:1148-1159.

6. Fleming MT, Morris MJ, Heller G, Scher HI. Post-therapy changes in PSA as an outcome measure in prostate cancer clinical trials. Nat Clin Pract Oncol. 2006;3:658-667.

7. Kuroda K, Liu H, Kim S, Guo M, Navarro V, Bander NH. Docetaxel down-regulates the expression of androgen receptor and prostate-specific antigen but not prostatespecific membrane antigen in prostate cancer cell lines: implications for PSA surrogacy. Prostate. 2009;69:1579-1585.

8. Scher HI, Morris MJ, Kelly WK, Schwartz LH, Heller G. Prostate cancer clinical trial end points: "RECIST"ing a step backwards. Clin Cancer Res. 2005;11: 5223-5232.

9. Price DT, Coleman RE, Liao RP, Robertson CN, Polascik TJ, DeGrado TR. Comparison of [18 F]fluorocholine and [18 F]fluorodeoxyglucose for positron emission tomography of androgen dependent and androgen independent prostate cancer. J Urol. 2002;168:273-280.

10. Oprea-Lager DE, van Kanten MP, van Moorselaar RJ, et al. $\left[{ }^{18} \mathrm{~F}\right]$ fluoromethylcholine as a chemotherapy response read-out in prostate cancer cells. Mol Imaging Biol. 2015;17:319-327.

11. De Giorgi U, Caroli P, Burgio SL, et al. Early outcome prediction on ${ }^{18} \mathrm{~F}$-fluorocholine PET/CT in metastatic castration-resistant prostate cancer patients treated with abiraterone. Oncotarget. 2014;5:12448-12458.

12. Miyazaki KS, Kuang Y, Kwee SA. Changes in skeletal tumor activity on ${ }^{18} \mathrm{~F}$ choline PET/CT in patients receiving ${ }^{223}$ radium radionuclide therapy for metastatic prostate cancer. Nucl Med Mol Imaging. 2015;49:160-164. 
13. Kwee SA, Lim J, Watanabe A, Kromer-Baker K, Coel MN. Prognosis related to metastatic burden measured by ${ }^{18} \mathrm{~F}$-fluorocholine PET/CT in castration-resistant prostate cancer. J Nucl Med. 2014;55:905-910.

14. Lim JL, Dorman ET, Cabral CL. Automated production of $\left[{ }^{18} \mathrm{~F}\right] \mathrm{FECh}$ and $\left[{ }^{18} \mathrm{~F}\right]$ FCH: preparation and use of $\left[{ }^{18} \mathrm{~F}\right]$ fluoroalkane sulfonates and fluoroalkylation agents [abstract]. J Label Compd Radiopharm. 2003;46:S46.

15. Bauman G, Belhocine T, Kovacs M, Ward A, Beheshti M, Rachinsky I. ${ }^{18} \mathrm{~F}-$ fluorocholine for prostate cancer imaging: a systematic review of the literature. Prostate Cancer Prostatic Dis. 2012;15:45-55.

16. De Giorgi U, Caroli P, Scarpi E, et al. ${ }^{18}$ F-fluorocholine PET/CT for early response assessment in patients with metastatic castration-resistant prostate cancer treated with enzalutamide. Eur J Nucl Med Mol Imaging. 2015;42:1276-1283.

17. Nelius T, Filleur S. PSA surge/flare-up in patients with castration-refractory prostate cancer during the initial phase of chemotherapy. Prostate. 2009;69: 1802-1807.

18. Halabi S, Vogelzang NJ, Ou SS, Owzar K, Archer L, Small EJ. Progression-free survival as a predictor of overall survival in men with castrate-resistant prostate cancer. J Clin Oncol. 2009;27:2766-2771.

19. Hussain M, Goldman B, Tangen C, et al. Prostate-specific antigen progression predicts overall survival in patients with metastatic prostate cancer: data from Southwest Oncology Group trials 9346 (intergroup study 0162) and 9916. J Clin Oncol. 2009;27:2450-2456.
20. Ceci F, Castellucci P, Graziani T, et al. ${ }^{11} \mathrm{C}$-choline PET/CT in castrationresistant prostate cancer patients treated with docetaxel. Eur J Nucl Med Mol Imaging. 2016;43:84-91.

21. Colombié M, Campion L, Bailly C, et al. Prognostic value of metabolic parameters and clinical impact of ${ }^{18} \mathrm{~F}$-fluorocholine PET/CT in biochemical recurrent prostate cancer. Eur J Nucl Med Mol Imaging. 2015;42:17841793.

22. Shah RB, Mehra R, Chinnaiyan AM, et al. Androgen-independent prostate cancer is a heterogeneous group of diseases: lessons from a rapid autopsy program. Cancer Res. 2004;64:9209-9216.

23. Odewole OA, Oyenuga OA, Tade F, et al. Reproducibility and reliability of anti$3-\left[{ }^{18} \mathrm{~F}\right] \mathrm{FACBC}$ uptake measurements in background structures and malignant lesions on follow-up PET-CT in prostate carcinoma: an exploratory analysis. Mol Imaging Biol. 2015;17:277-283.

24. Szabo Z, Mena E, Rowe SP, et al. Initial evaluation of $\left[{ }^{18} \mathrm{~F}\right] \mathrm{DCFPyL}$ for prostatespecific membrane antigen (PSMA)-targeted PET imaging of prostate cancer. Mol Imaging Biol. 2015;17:565-574.

25. Yu EY, Duan F, Muzi M, et al. Castration-resistant prostate cancer bone metastasis response measured by ${ }^{18} \mathrm{~F}$-fluoride PET after treatment with dasatinib and correlation with progression-free survival: results from American College of Radiology Imaging Network 6687. J Nucl Med. 2015;56: 354-360. 